\title{
Employing Photoassisted Ligand Exchange Technique in Layered Quantum Dot LEDs
}

\author{
Wenjia Hu, ${ }^{1,2}$ Shuai Gao,, ${ }^{1,2}$ Paras N. Prasad, ${ }^{3}$ Jingkang Wang, ${ }^{2}$ and Jian $\mathrm{Xu}^{1}$ \\ ${ }^{1}$ Chemical Engineering and Technology, Tianjin University, Tianjin 300072, China \\ ${ }^{2}$ Engineering Science and Machines, The Pennsylvania State University, University Park, PA 16802, USA \\ ${ }^{3}$ Institute for Lasers, Photonics and Biophotonics, University at Buffalo, The State University of New York, Buffalo, NY 14260, USA \\ Correspondence should be addressed to Jian Xu, jianxu@engr.psu.edu
}

Received 28 August 2011; Accepted 31 October 2011

Academic Editor: Fan Zhang

Copyright (c) 2012 Wenjia Hu et al. This is an open access article distributed under the Creative Commons Attribution License, which permits unrestricted use, distribution, and reproduction in any medium, provided the original work is properly cited.

We presented in this paper a photoassisted ligand exchange approach whereby light will be introduced to facilitate the replacement of oleic acid (OA) ligand molecules over PbSe quantum dots (QDs). The ligand-exchanged QDs were used to fabricate quantum dot light-emitting-diodes (QD-LEDs), which outperform the devices comprising the QDs without ligand-replacement.

\section{Introduction}

Colloidal semiconductor quantum dots (QDs), by virtue of the large tunability in their bandgap, high luminance efficiency, narrow spectral emission, and high photostability, are promising lumophores and sensitizers in next-generation optoelectronic devices, such as light emitting diodes (LEDs) [1], photo detectors [2], and solar cells $[3,4]$. However, the surface of the QDs is often capped with long-chain ligand molecules, which are electrically insulating and thus hinder the electronic applications of the QDs [5]. Ligand exchange with shorter and more conductive surfactant molecules is necessary for improved device performance (Our work (Nanotechnology) or D.Sargent's work). we reported in this communication the use of a photoassisted ligand exchange approach to facilitate the dot-to-dot electron transport, which results in a significant improvement in the output emission of QD-LEDs.

\section{Experiment Details}

PbSe QDs were synthesized following the noncoordinating solvent technique developed by Yu et al. [6]. The as prepared PbSe QDs, $4.5 \mathrm{~nm}$ in diameter and nearly monodisperse, were stabilized with a capping layer of oleate molecules coordinated to the $\mathrm{Pb}$ atoms (Figure 1). Ligand exchange processing of the PbSe QDs was based on the method proposed by Won et al. [7]. $3 \mathrm{~mL}$ tert-butyl N-(2mercaptoethyl)carbamate(t-BOC) and $15 \mathrm{mg} / \mathrm{mL}$ PbSe QDs in hexane solution $(5 \mathrm{~mL})$ were mixed in centrifuge tubes, and then vortexed overnight under nitrogen. Because the $-\mathrm{S}-\mathrm{R}_{n}$ in $\mathrm{t}-\mathrm{BOC}$ has higher polarity than $-\mathrm{O}-\mathrm{CO}-\mathrm{R}_{n}$ in oleic acid, the surface ligands of PbSe QDs will be replaced by tBOC. Following ligand replacement, the QDs were washed twice with methanol using a centrifuge and then redispersed in chloroform. By this simple treatment the 18-carbon-chain oleate molecules overcoating the PbSe QDs were replaced with 4-carbon-chain 2-Mercaptoethylamine surfactants.

The design of the QD-LEDs was illustrated in Figure 2. A multilayer structure was employed in the device fabrication, consisting of ITO/poly(3,4-ethylenedioxythiophene) poly (styrenesulfonate) (PEDOT/PSS) $(25 \mathrm{~nm}) /$ hole transport layer (HTL) $(45 \mathrm{~nm}) / 2$-Mercaptoethylamine capped PbSe QD ( $\sim 3$ monolayers)/electron transport layer(ETL) $(50 \mathrm{~nm}) / \mathrm{Al}(150 \mathrm{~nm})$. In the device, ITO/PEDOT/PSS was used as the anode. The HTL was solution-cast deposited from poly-(N, $\mathrm{N}^{\prime}$-bis(4-butylphenyl)-N, $\mathrm{N}^{\prime}$-bis(phenyl) benzidine) (poly-TPD) in chlorobenzene solution. The emissive layer of the device contains the mixture of ligandexchanged QDs and PAG (di-tert-butylphenyliodonium perfluorobutanesulfonate) that was spin deposited from the chloroform solution. Processing of the emissive layer also involves the consecutive UV exposure $(265 \mathrm{~nm}, 5 \mathrm{mins})$ and annealing at $120^{\circ} \mathrm{C}$ for an extended period. Tris 


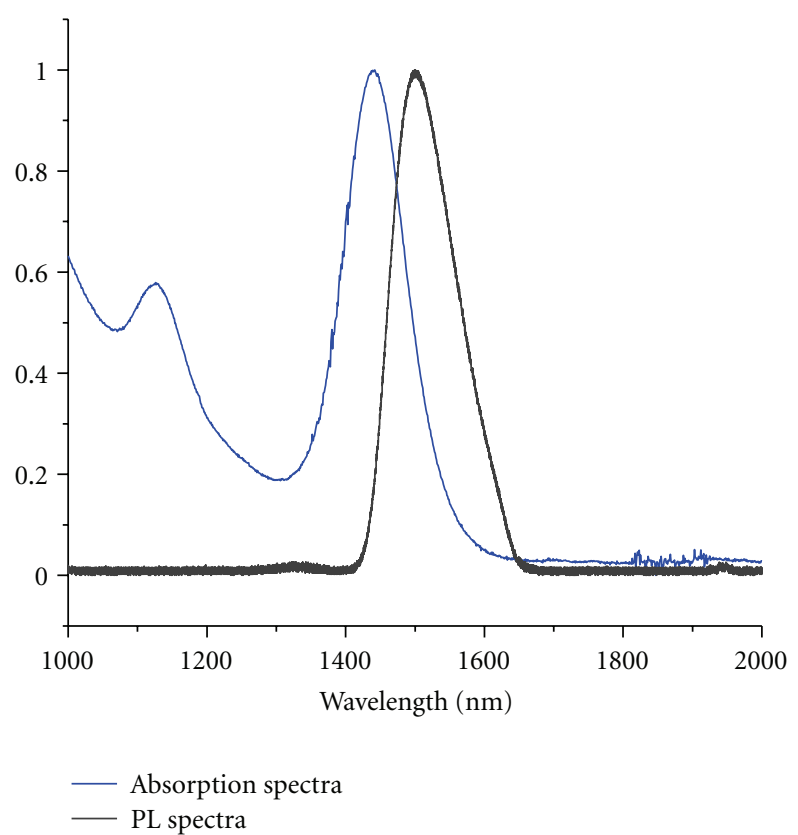

(a)

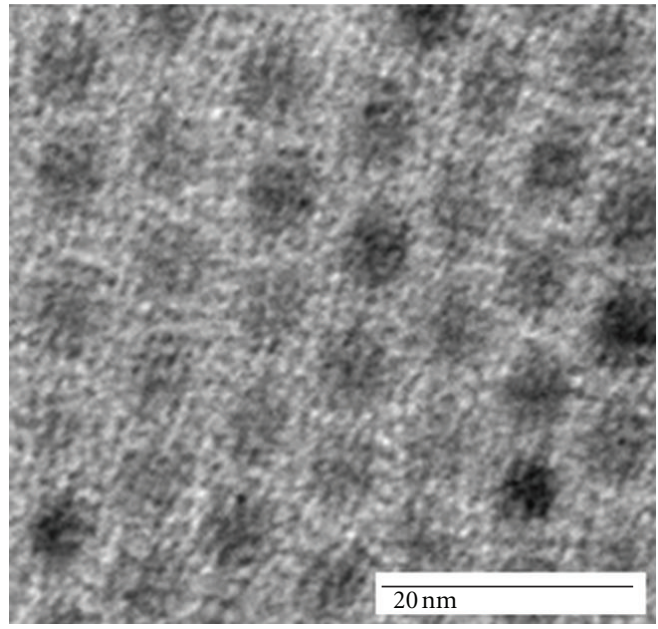

(b)

FIGURE 1: (a) Absorption and photoluminous spectra of as prepared PbSe QDs; (b) TEM image of PbSe QDs with an average diameter of $4.5 \mathrm{~nm}$.

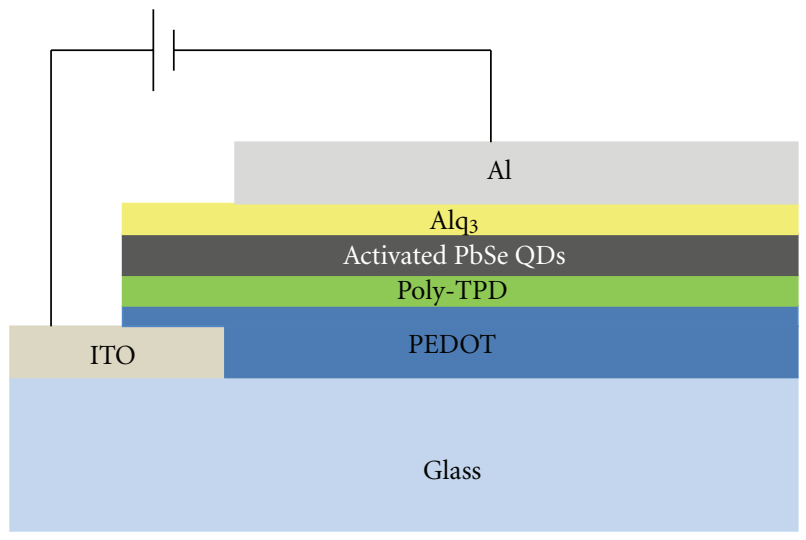

Figure 2: Schematic of a PbSe QD-LED device structure.

(8-hydroxyquinoline) aluminum ( $\left.\mathrm{Alq}_{3}\right)$ was evaporated over the QD layer as the ETL, and the shadow mask-evaporated aluminum film was used as the cathode.

The absorption spectra of the QD solution and films were recorded using a Perkin-Elmer Lambda 19 UV/VIS/NIR spectrometer. Photoluminescence (PL) spectra were measured by a CM110 Spectral-Products NIR spectrometer using a $980 \mathrm{~nm}$ diode laser module as the excitation source. The absorption and PL spectra were recorded at room temperature. The FTIR spectra of the QDs before and after ligand exchange were measured using a Bruker IFS 66/S FT-IR Spectrometer, A JEOL FasTEM-2010 transmission electron microscope (TEM) was used to image the crystal lattice of the QDs.

\section{Result and Discussion}

3.1. Surface Treatment of PbSe QDs. The PbSe QDs were stabilized with a capping layer of oleate molecules coordinated to the $\mathrm{Pb}$ atoms [8]. The 18-carbon long-chain structure renders the oleate molecules insulating and hinders the dot-to-dot transport of the free carriers in the QDs. Figure 3 illustrates the principle of changing the oleate molecules to the ones of shorter molecular chains and better conductivity: after initial treatment of $\mathrm{t}$-BOC, the oleic acid ligands overcoating the PbSe QDs were replaced by t-BOC. Figure 4(a) displays the FTIR spectra before and after ligand exchange. Significant changes are clearly observed between the t-BOC-protected QDs and the oleic acid-capped QDs. 


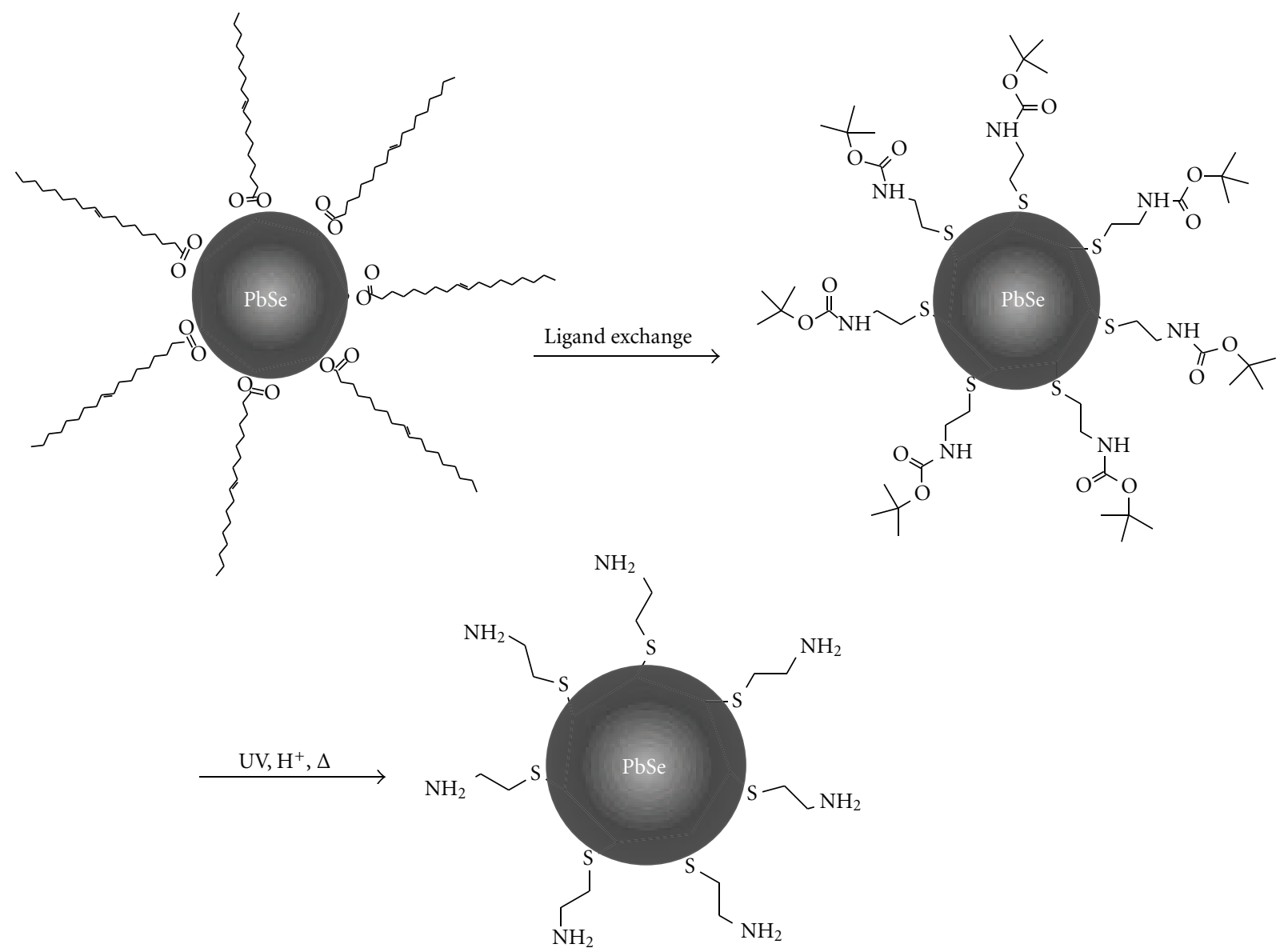

FIGURE 3: Scheme of ligand exchange process.

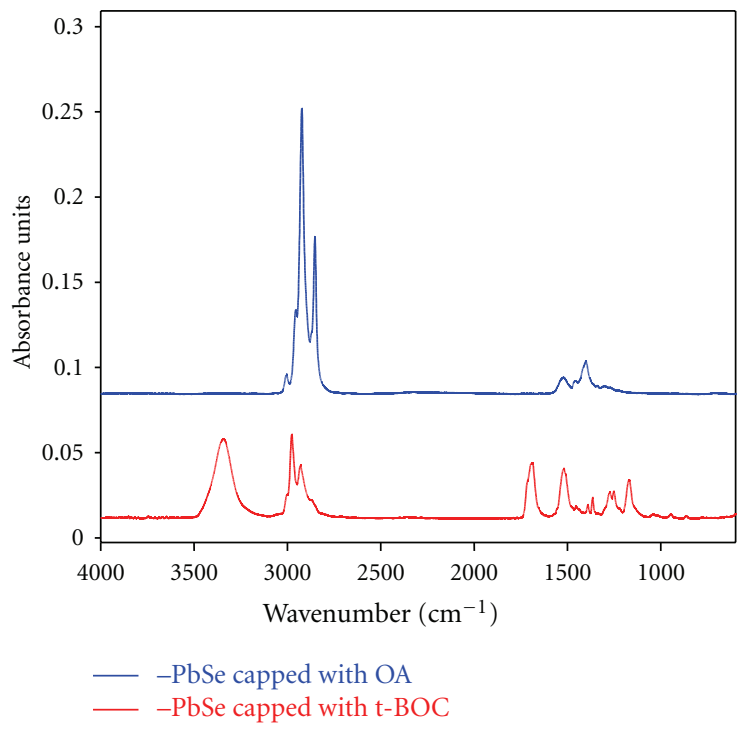

(a)

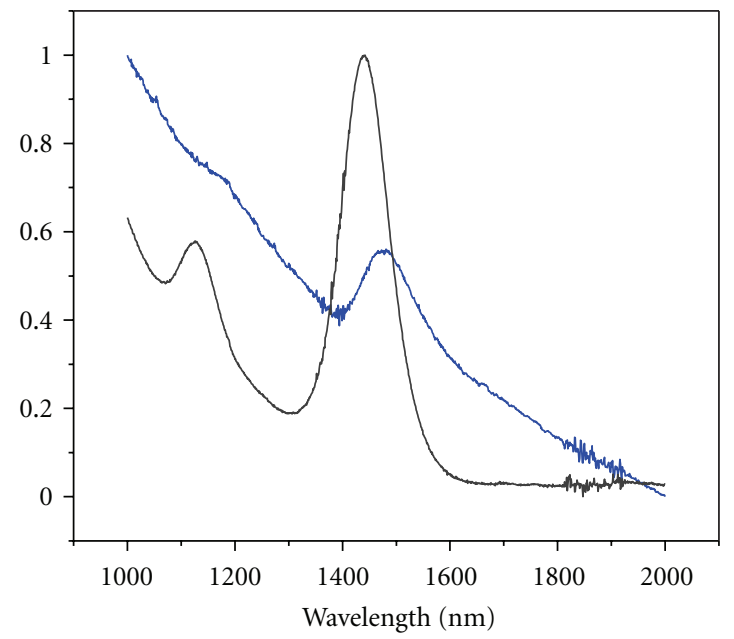

- Oleic acid capped QDS

- Tert-butyl N-(2-mercaptoethyl) carbamate capped QDs

(b)

FIGURE 4: (a) FTIR spectra before and after ligand exchange. (b) Absorption spectra before and after ligand exchange. 


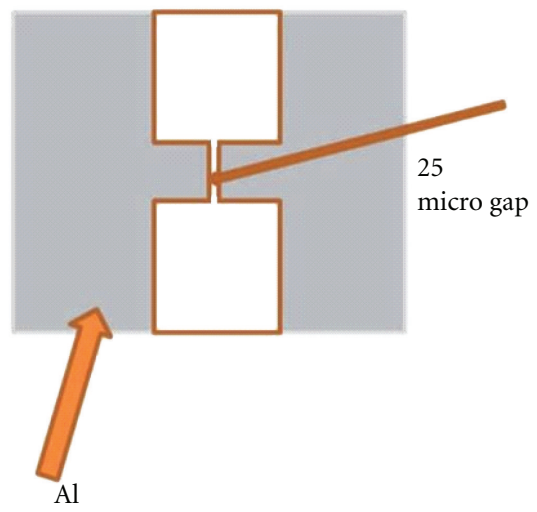

(a)

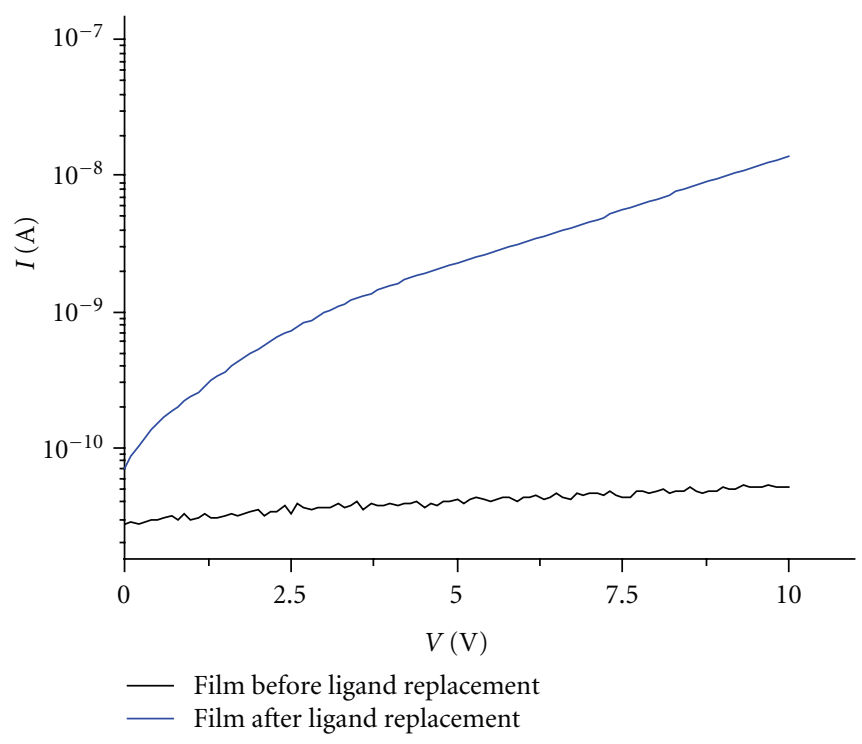

(b)

FIGURE 5: (a) The structure of the conductivity measurement. (b) I-V curve of the QDs before and after ligand exchange.

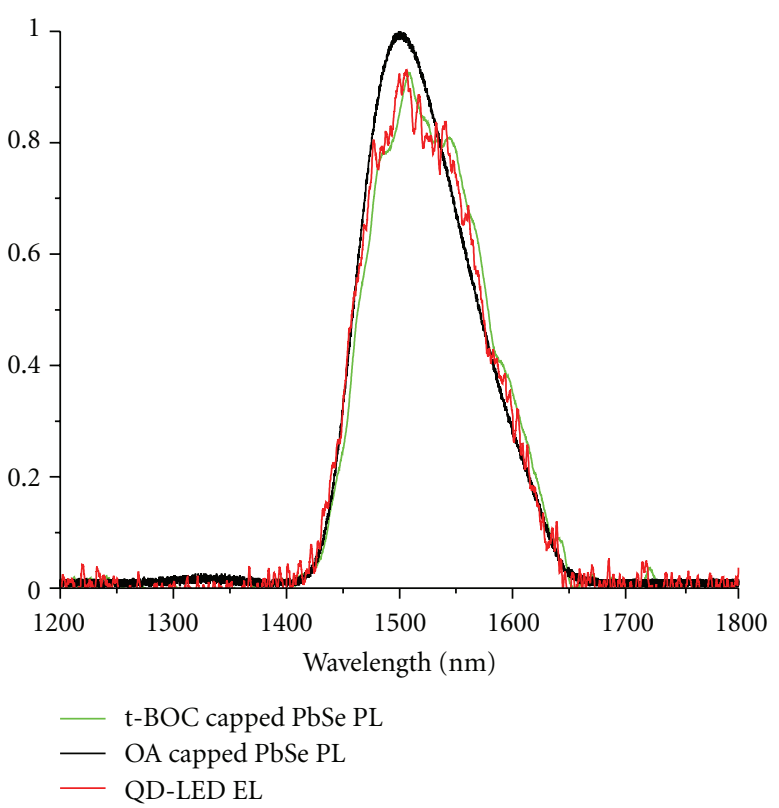

(a)

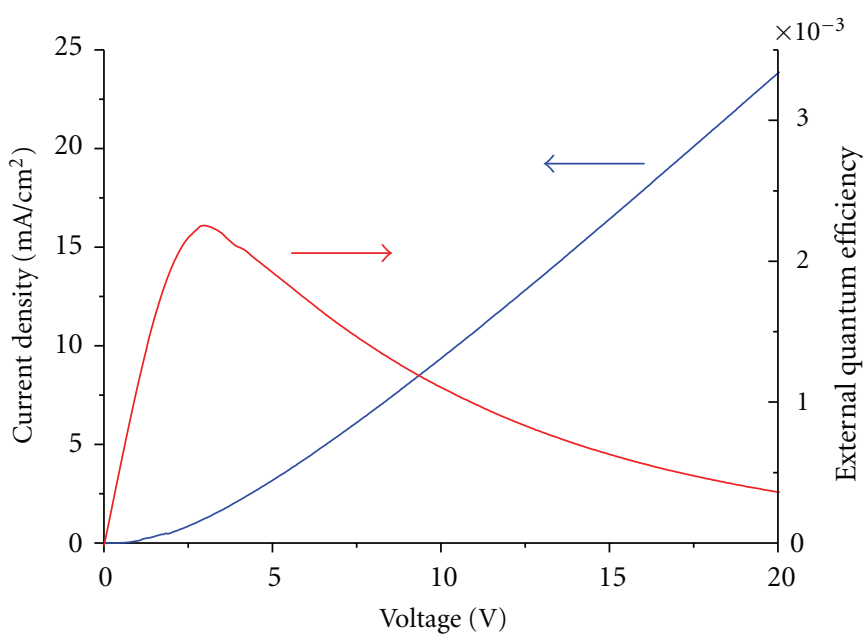

(b)

FIgure 6: (a) PL spectra of the OA capped PbSe QDs (black) and the ligand-exchanged QDs (green); and EL spectrum of the QD-LED (red), (b) external quantum efficiency and current voltage plot for a PbSe QD-LED.

Specifically, the vibrational peak of the carbonyl hydrogen bonds below $3000 \mathrm{~cm}^{-1}$ decrease drastically as the long carbon chain was replaced by shorter carbon chain. The absorption peak at $3350 \mathrm{~cm}^{-1}$ arises, which can be attributed to the $-\mathrm{NH}$ vibrations of t-BOC. Figure 4(b) shows the absorption spectra of the QD solution. There is a slight red shift after ligand exchange due to the enhanced Stark effect that was induced by the variation of the surface-polarization energy during ligand replacement [9]. The new ligand $\mathrm{t}-\mathrm{BOC}$ was unstable. By reacting with the photochemically generated acid under UV irradiation, the chemical bond between $-\mathrm{NH}$ and $-\mathrm{C}=\mathrm{O}$ cracked. Finally, the PbSe QDs were capped with 2-Mercaptoethylamine of a substantially reduced molecular chain length.

To measure the conductivity before and after ligand exchange, a gapped electrode structure was fabricated using fine gold filament as the shadow mask, as showed in Figure 5(a); PbSe QDs was drop-cast on top of the electrode gap 
for the conduction measurement. Figure 5(b) compares the results of the conduction measurement for the samples with and without ligand exchange, showing that the conductivity of the ligand-exchanged PbSe QDs increased remarkably. This suggests that the ligand molecular length plays a key role for the carrier transport between the QDs under study.

3.2. PbSe QD-LED Characteristics. The NIR electroluminescence (EL) spectrum of the QD-LED closely resembles the photoluminescence (PL) of the ligand exchanged QDs as showed in Figure 6(a). It is noteworthy that the PL profile of the ligand-exchanged QDs exhibits a slight red shift from that of the as prepared PbSe QDs, which is consistent with the absorption spectra discussed previously.

For the sake of comparison, we have fabricated LED control samples using the PbSe QDs overcoated with oleate molecules. We did not observe any EL signal from the control samples. It is thus evident from our study that carrier transport across the QD surface was dramatically improved following the photoassisted ligand exchange process, which, in turn, leads to the enhanced injection and radiative recombination of electrons and holes in the nanoparticles. These experimental results proved that the ligand exchange method of the PbSe QDs strongly enhanced the injection and radiative recombination of electron and holes in the nanoparticle QDs in layered QD-LEDs. The accurate measurement of the QD-LED followed Steckel's method [10], the power efficiency of this device is no better than $0.1 \%$ (Figure 6(b)), implying that $99.9 \%$ of the power was converted to heat, while a large portion of the heat will be lost to the surrounding air by conduction and conversion process.

\section{Conclusion}

A photoassisted ligand exchange approach was employed to replace the oleic acid ligand on the surface of the QDs. The length of the carbon chain was successfully decreased by the photochemical reaction. Consequently, carrier transport across the QD surface was significantly enhanced. The surface-treated QDs were used to fabricate thin-film QDLEDs, which results in improved LED performance over the untreated QD-LEDs.

\section{Acknowledgments}

The work at The Pennsylvania State University is being supported by the National Science Foundation under Grants of CMMI-0729263 and ECCS-0824186 and Army Research Office under Grant nos. 49653-EL and DURIP 2008-02-136.
[3] I. Gur, N. A. Fromer, M. L. Geier, and A. P. Alivisatos, "Air-stable all-inorganic nanocrystal solar cells processed from solution," Science, vol. 310, no. 5747, p. 462, 2005.

[4] Z. Tan, T. Zhu, M. Thein et al., "Integration of planar and bulk heterojunctions in polymer/nanocrystal hybrid photovoltaic cells," Applied Physics Letters, vol. 95, no. 6, Article ID 063510, 2009.

[5] M. Law, J. M. Luther, Q. Song, B. K. Hughes, C. L. Perkins, and A. J. Nozik, "Structural, optical, and electrical properties of PbSe nanocrystal solids treated thermally or with simple amines," Journal of the American Chemical Society, vol. 130, no. 18, pp. 5974-5985, 2008.

[6] W. W. Yu, J. C. Falkner, B. S. Shih, and V. L. Colvin, "Preparation and characterization of monodisperse PbSe semiconductor nanocrystals in a noncoordinating solvent," Chemistry of Materials, vol. 16, no. 17, pp. 3318-3322, 2004.

[7] J. K. Won, J. K. Sung, K. S. Lee, M. Samoc, A. N. Cartwright, and P. N. Prasad, "Robust microstructures using UV photopatternable semiconductor nanocrystals," Nano Letters, vol. 8, no. 10, pp. 3262-3265, 2008.

[8] I. Moreels, B. Fritzinger, J. C. Martins, and Z. Hens, "Surface chemistry of colloidal PbSe nanocrystals," Journal of the American Chemical Society, vol. 130, no. 45, pp. 15081-15086, 2008.

[9] Y. Zhang, Q. Dai, X. Li et al., "Formation of PbSe/CdSe Core/Shell nanocrystals for stable near-infrared high photoluminescence emission," Nanoscale Research Letters, vol. 5, no. 8, pp. 1279-1283, 2010.

[10] J. S. Steckel, S. Coe-Sullivan, V. Bulović, and M. G. Bawendi, " 1 . $\mathrm{m}$ to $1.55 \mathrm{~m}$ tunable electroluminescence from PbSe quantum dots embedded within an organic device," Advanced Materials, vol. 15, no. 21, pp. 1862-1866, 2003.

\section{References}

[1] Q. Sun, Y. A. Wang, L. S. Li et al., "Bright, multicoloured lightemitting diodes based on quantum dots," Nature Photonics, vol. 1, no. 12, pp. 717-722, 2007.

[2] D. Qi, M. Fischbein, M. Drndić, and S. Šelmić, "Efficient polymer-nanocrystal quantum-dot photodetectors," Applied Physics Letters, vol. 86, no. 9, Article ID 093103, 2005. 

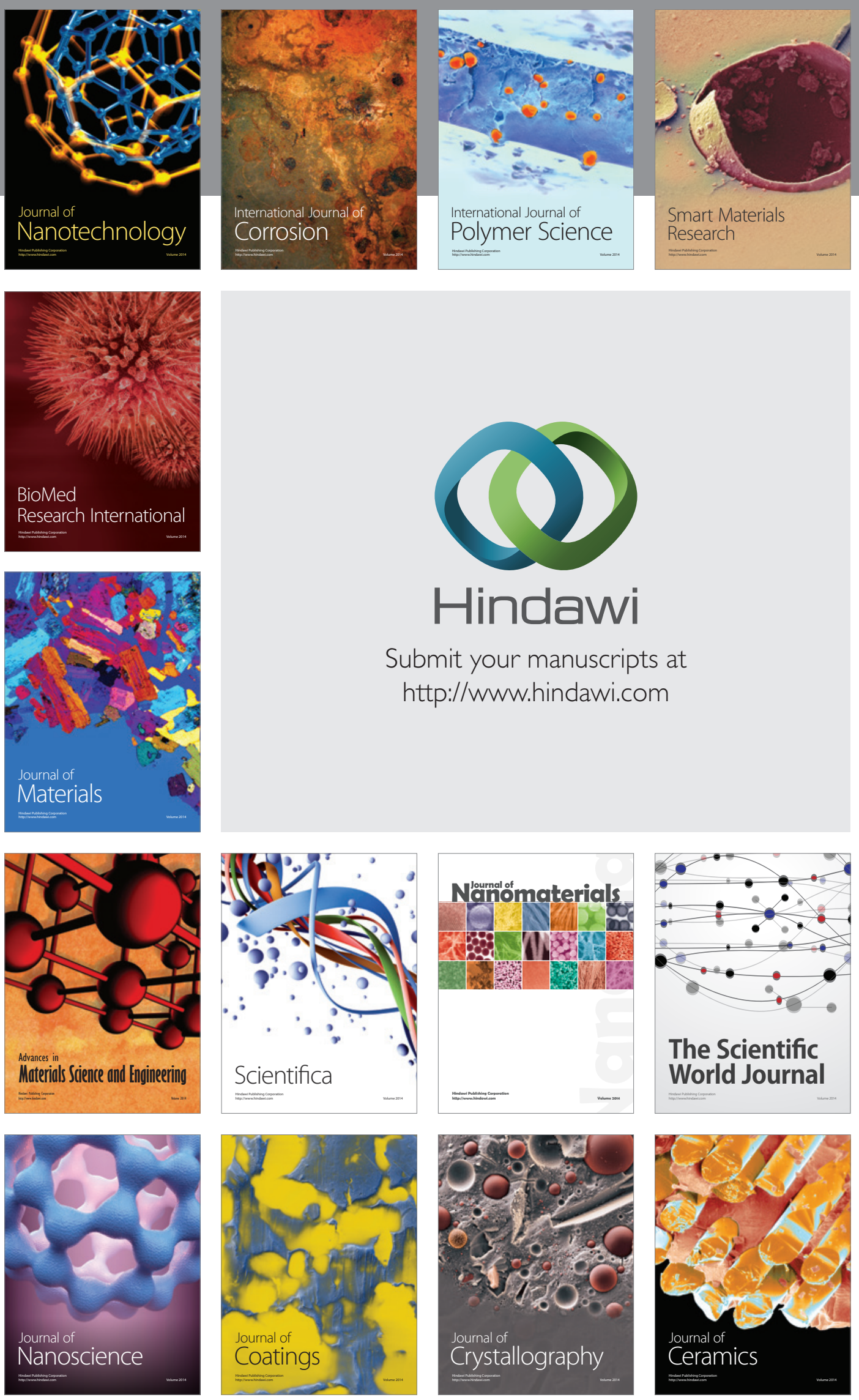

The Scientific World Journal

Submit your manuscripts at

http://www.hindawi.com

\section{World Journal}

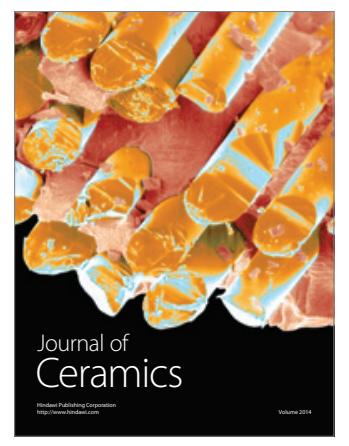

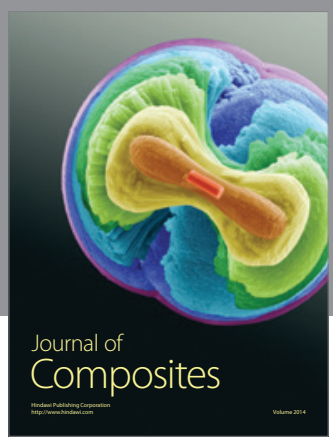
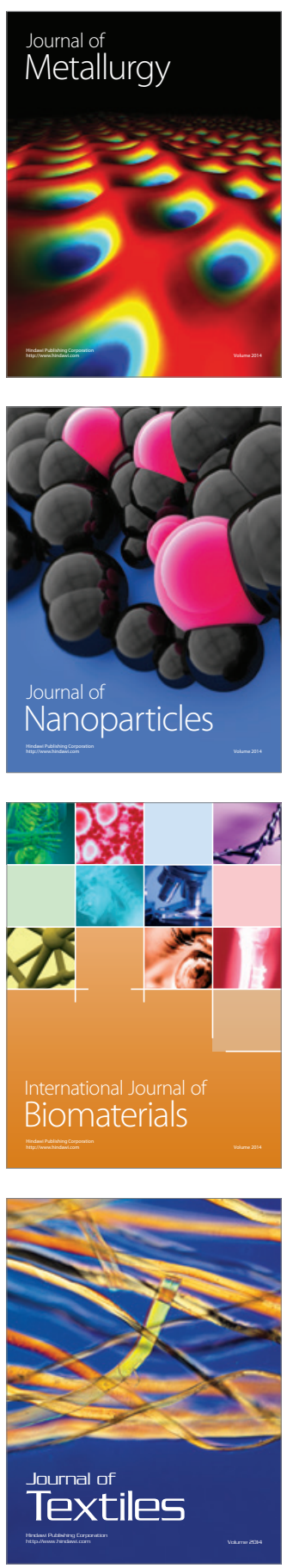